\title{
COMA HIPEROSMOLAR ASSOCIADO AO TRANSPLANTE DE FÍGADO
}

\author{
Hyperosmolar coma associated with liver transplantation
}

\author{
Olival Cirilo Lucena da FONSECA-NETO, Cláudio Moura LACERDA
}

ABCDDV/XX

Fonseca-Neto OCL, Lacerda CM. Coma hiperosmolar associado ao transplante de fígado. ABCD Arq Bras Cir Dig 2009;22(1):60-1

RESUMO - Introdução - O diagnóstico diferencial dos pacientes inconscientes sempre inclui o coma hiperosmolar hiperglicêmico não-cetótico. Relato de caso - Paciente do sexo feminino, 22 anos, tipo sangüíneo $\mathrm{O}+$, branca, natural e procedente do Recife - PE com queixa de icterícia e astenia há um mês. Ao exame físico, havia icterícia 3+/4+, desnutrição leve (IMC 17,5) e asterixis. Os exames laboratoriais sugeriram hepatite fulminante. Após 12 horas da inclusão na lista de espera pelo transplante, recebeu enxerto de fígado de doador cadáver, mediante o uso de técnica de piggback sem intercorrências. No $10^{\circ}$ dia pós-operatório evoluiu com trombose de artéria hepática (TAH), diagnosticada por ultra-som com doppler de artéria hepática. Após 48 horas foi retransplantada sem intercorrências. A partir do $13^{\circ}$ dia de pós-operatório, evoluiu com hiperglicemia grave (> $600 \mathrm{mg} / \mathrm{dl}$ ) e alteração do nível de consciência (9 pontos na escala de coma de Glasgow). Osmolaridade sérica nesse momento igual a $309 \mathrm{mOsm} /$ $\mathrm{kgH} 2 \mathrm{O}$. O nível sérico de tacrolimus nesse dia foi de $11 \mathrm{ng} / \mathrm{dl}$. Coma hiperosmolar hiperglicêmico não-cetótico foi tratado clinicamente por 48 horas. Recebeu alta no $30^{\circ}$ dia de pós-operatório do retransplante, sem diabete mellitus. Conclusão - O coma hiperosmolar é um evento raro no pós-operatório de transplante de fígado e pode modificar a evolução do paciente.

DESCRITORES - Transplante de fígado. Coma hiperosmolar. Hiperglicêmico.

\section{INTRODUÇÃO}

A hiperglicemia é evento comum no pós-operatório precoce de transplante hepático, em razão de vários fatores, inclusive as profundas alterações no ambiente hormonal do organismo. Altos valores de glicemia podem ser causados por infecção, complicações de imunossupressão ou nutrição parenteral. $\mathrm{O}$ estado de coma hiperglicêmico hiperosmolar não-cetótico (CHHNC) é uma grave complicação do metabolismo da glicose, e é raramente descrito no pós-operatório de transplante de fígado. Os autores descrevem caso de CHHNC no pós-operatório precoce de transplante de fígado, em paciente não diabética, e com evolução favorável após o início do tratamento.

\section{RELATO DE CASO}

Paciente do sexo feminino, 22 anos, tipo sangüíneo $\mathrm{O}+$, branca, natural e procedente do Recife - PE. Encaminhada ao Serviço de Cirurgia Geral e Transplante de Fígado do Hospital Universitário Oswaldo Cruz em julho

Trabalho realizado no Serviço de Cirurgia Geral e Transplante de Fígado do Hospital Universitário Oswaldo Cruz, Universidade de Pernambuco.

Correspondência: Dr. Olival Cirilo Lucena Fonseca Neto. E-mail: olivalneto@globo.com de 2004 com queixa de icterícia e astenia há um mês. Ao exame físico, havia icterícia $3+/ 4+$, desnutrição leve (IMC 17,5) e asterixis. Os exames laboratoriais constam da tabela 1. A paciente recebeu o diagnóstico de hepatite fulminante que mais tarde foi confirmada ser secundária a Doença de Wilson. Após 12 horas da inclusão na lista de espera pelo transplante, recebeu enxerto de fígado de doador cadáver, mediante o uso de técnica de piggback sem intercorrências. Apresentou pós-operatório imediato sem grandes alterações. No $10^{\circ}$ dia pós-operatório evoluiu com trombose de artéria hepática (TAH), diagnosticada por ultra-som com doppler de artéria hepática. A paciente apresentou piora progressiva do quadro clínico e após 48 horas do diagnóstico, recebeu novo enxerto de fígado proveniente de doador cadáver, procedimento realizado segundo técnica piggyback. A partir do $13^{\circ}$ dia de pósoperatório, evoluiu com hiperglicemia grave (> $600 \mathrm{mg} / \mathrm{dl})$ e alteração do nível de consciência ( 9 pontos na escala de coma de Glasgow). Osmolaridade sérica nesse momento igual a $309 \mathrm{mOsm} / \mathrm{kgH} 2 \mathrm{O}$. O nível sérico de tacrolimus nesse dia foi de $11 \mathrm{ng} / \mathrm{dl}$. Hemoculturas, culturas de urina e ponta de cateter foram negativas. Correção dos distúrbios hidrossalinos, diminuição da imunossupressão e de diuréticos, reajuste da alimentação enteral e introdução de antimicrobianos foram os meios utilizados para o tratamento da síndrome metabólica. Não houve necessidade de intubação orotraqueal. Após 48 horas, a paciente evoluiu 
com melhora clínica e laboratorial. Recebeu alta no $30^{\circ}$ dia de pós-operatório do retransplante, sem diabete mellitus.

TABELA 1 - Exames laboratoriais no momento da admissão hospitalar

\begin{tabular}{ll}
\hline Bilirrubinas totais / Bilirrubinas diretas & \multicolumn{1}{c}{ 29mg/d / 22mg/dl } \\
\hline INR & 6 \\
AST & $214 \mathrm{U} /$ litro \\
ALT & $148 \mathrm{U} /$ litro \\
FA & $471 \mathrm{U} /$ litro \\
GGT & $124 \mathrm{U} /$ litro \\
DHL & $287 \mathrm{U} /$ litro \\
PLAQ & $51.000 / \mathrm{mm} 3$ \\
\hline
\end{tabular}

\section{DISCUSSÃO}

A síndrome do coma hiporosmolar hiperglicêmico não-cetótico (CHHNC) foi descrita por Sament e Schwartz em 1957 em paciente diabético ${ }^{3}$. Consiste em grave desequilíbrio do estado hidrossalino e osmolar. Se não precocemente diagnosticado, pode ter má evolução. O CHHNC é complicação pouco freqüente em pacientes não-diabéticos. As complicações metabólicas após transplante ortotópico de fígado (TOF) apresentam incidências semelhantes a do transplante renal, e são diretamente associadas a imunossupressão ${ }^{1}$. Em pacientes transplantados renais, a incidência de $\mathrm{CHHNC}$ em paciente não diabético é de 1.1/1000 pacientes/ano. O fígado é o principal órgão responsável pelo metabolismo da glicose e alterações do metabolismo da glicose em pacientes submetidos a ressecções hepáticas e TOF são esperadas. Nos primeiros pacientes submetidos transplantes de fígado, alguns casos de hiperglicemia grave $(>1200 \mathrm{mg} / \mathrm{dl})$ foram observados ${ }^{4}$. No entanto, esses valores estavam ligados ao tratamento metabólico adotado. CHHNC foi relatado em paciente de 11 anos de idade, após transplante de fígado ${ }^{2}$. Ele apresentou CHHNC (glicemia em torno de $630 \mathrm{mg} / \mathrm{dl}$ ) e evoluiu para o óbito. Várias causas estão associadas ao aparecimento da síndrome: altas doses de corticóides, níveis séricos elevados de tacrolimus, infecção, uso excessivo de diuréticos, hiperalimentação venosa, nutrição enteral hiperprotéica e hiperosmolar. A mortalidade pode ser tão alta quanto 50\% dos casos, e é mais alta se choque séptico também estiver presente. Consiste em grave evento, associado ao aumento da mortalidade dos pacientes transplantados ${ }^{5}$. $\mathrm{O}$ tratamento baseia-se na hidratação criteriosa, uso de insulina, correção dos distúrbios iônicos e busca de infecção em paciente imunossuprimido. O diagnóstico precoce, e o tratamento adequado podem mudar o prognóstico do quadro clínico.

\section{CONCLUSÃO}

O coma hiperglicêmico hiperosmolar não-cetótico é uma complicação metabólica não usual em receptores de transplante hepático e que pode levar ao óbito.

Fonseca-Neto OCL, Lacerda CM. Hyperosmolar coma associated with liver transplantation. ABCD Arq Bras Cir Dig 2009;22(1):60-1

ABSTRACT - Background - The literature described an increased incidence of gastrointestinal stromal tumors (GISTs) in patients with neurofibromatosis type 1 . These tumors typically occur in the small intestine, and frequently are multiple. Often the behavior of the tumor in this association is more favorable than in sporadic cases. Case report - Incidental diagnosis of GIST was done in a patient with neurofibromatosis type 1 during treatment for acute abdomen. Trans-operatively was identified a retrocecal perforated appendicitis and a neoplastic mass in the proximal jejunum. The lesion occupied approximately $70 \%$ of the circumference of the organ and had no invasion of adjacent structures. Moreover, there were dozens of small nodules scattered throughout the length of the jejunum and ileum. The pathology revealed malignancy consistent with GIST, with moderate degree of atypia, low mitotic index $(<5 / 50)$ and absence of necrosis. In immunohistochemical analysis, the neoplastic cells were positive for CD-34 and CD-117 (c-KIT), and negative for desmin. After surgery, the use of Imatinib chemotherapy was indicated. After a follow-up period of 12 months, the patient showed no signs of recurrence. Conclusion - GISTs should be considered in the presence of abdominal mass and neurofibromatosis type 1, affecting mainly small bowel. Sometimes Imatinib can be administered with good results in the control of the disease.

HEADINGS - Neurofibromatosis. Gastrointestinal stromal tumors. Acute abdomen.

\section{REFERÊNCIAS}

1. Lampe EW, Ruiz JO, Simmons RL, Najarian JS. Hyperglicemic nonketotic coma after renal transplantation. Am J Surg 1974; 127:342-44.

2. Machado MCC, Monteiro da Cunha JE, Margarido NF, Bacchella T, Lima Gonçalves E, Raia AA. Hyperosmolar coma associated with clinical liver transplantation. Int Surg 1976; 61:368.

3. Sament S, Schwartz MB. Severe diabetic stupor without ketosis. S Afr Med J 1957; 54:683-699.

4. Starzl TE, Putnam CW. Experience in hepatic transplantation. Philadelphia,
WB Saunders Co 1969, pp 92-98.

5. Van den Berghe G, Wouters PJ, Bouillon R, Weekers F, Verwaest C, Schetz, Vlasselaers D, Ferdinande P, Lauwers P. Outcome benefit of intensive insulin therapy in the critically ill: insulin dose versus glycemic control. Crit Care Med 2003; 31(2):359-66

Fonte de financiamento: não há Conflito de interesse: não há Recebido para publicação: 08/08/2008 Aceito para publicação: 14/12/2008 